\title{
Estudio del Efecto de Reductores de Viscosidad en Crudo Pesado
}

\section{Effect of Viscosity Reducers on Heavy Crude Oil}

\author{
Alex Abarca \\ Escuela Politécnica Nacional \\ Quito, Ecuador \\ alex.abarca@epn.edu.ec \\ Orcid: 0000-0003-3796-2652
}

\author{
Liliana Guzmán-Beckmann \\ Escuela Politécnica Nacional \\ Quito, Ecuador \\ liliana.guzman@epn.edu.ec \\ Orcid: 0000-0003-1623-4015
}

\author{
Jessica Castillo \\ Escuela Politécnica Nacional \\ Quito, Ecuador \\ jessica.castillo@epn.edu.ec \\ Orcid: 0000-0002-7047-5613
}

\begin{abstract}
Resumen - El crudo pesado, por su alta viscosidad, requiere de mayor energía para su transporte debido a que produce disminución en la eficiencia de las bombas de transferencia. En esta investigación, se evaluó el efecto de productos reductores de viscosidad a nivel de laboratorio y en pruebas de campo. En los ensayos de laboratorio se probaron cuatro tipos de reductores de viscosidad en un crudo del oriente ecuatoriano y se elaboraron curvas de temperatura de calentamiento y enfriamiento de 40 a $80^{\circ} \mathrm{C}$ con dosificaciones de 400, 550, 700 y 1000 ppm. El crudo utilizado en el estudio presentó $38 \%$ BS\&W y $19,5^{\circ} \mathrm{API}$. El producto que entregó los mejores resultados fue el denominado VRX-02, con dosificación de $700 \mathrm{ppm}$. Las pruebas en campo se realizaron para evaluar la eficiencia del aditivo VRX-02, se inyectó este producto en una línea de transferencia de $15 \mathrm{~km}$. Se obtuvo una reducción de viscosidad del crudo del $28 \%$ a la temperatura operación de $30^{\circ} \mathrm{C}$. Se registró un incremento en la transferencia de 198 barriles de fluido total (agua y crudo) por día (BFPD) y un incremento de crudo seco de 249 barriles por día (BOPD), debido a que el químico actúa también como rompedor de emulsión y permite una mejor separación del crudo y el agua. Con los resultados obtenidos se realizó el diseño de un sistema de inyección del aditivo y se evaluó económicamente el proyecto. A través de indicadores financieros se determinó que la instalación del sistema de reducción de viscosidad sea favorable.
\end{abstract}

Palabras Clave: Viscosidad, reductores de viscosidad, crudo pesado, asfaltenos, inhibidor de asfaltenos.
Abstract - Due to its high viscosity, heavy crude oil requires more energy for transportation because it causes a decrease in the efficiency of transfer pumps. In this investigation, the effect of viscosity-reducing products was evaluated at laboratory level and on field tests. In laboratory tests, four types of viscosity reducers were tested on crude oil from the eastern region of Ecuador. Heating and cooling temperature curves of 40 to $80^{\circ} \mathrm{C}$ were prepared with dosages of $400,550,700$ and $1,000 \mathrm{ppm}$. The crude used in the study presented $38 \% \mathrm{BS} \& \mathrm{~W}$ and $19.5^{\circ} \mathrm{API}$. The best results were obtained with a dosage of $700 \mathrm{ppm}$ of VRX-02. Onside tests were carried out to evaluate the efficiency of the VRX02 additive. This product was injected into the $15 \mathrm{~km}$ transfer line. Oil viscosity decreased $28 \%$ at operating temperature $\left(30^{\circ} \mathrm{C}\right)$. There was an increase in oil transfer of 198 barrels of total fluid per day (BFPD), and an increase in dry crude of 249 barrels of crude per day (BOPD) because the chemical also acts as an emulsion breaker and allows a better separation of crude oil and water. With the results obtained, the design of an additive injection system was carried out and the project was economically evaluated. Through financial indicators, it was determined that the installation of the viscosity reduction system is favorable.

Keywords: Viscosity, viscosity reduction, heavy crude oil, asphaltenes, asphaltenes inhibitor.

Sumario: I Introducción, II Metodología, III Resultados y Discusión, IV Conclusiones.

Como citar: Abarca, Alex., Guzmán-Beckmann, Liliana., Castillo, Jessica., (2020). Estudio del Efecto de Reductores de Viscosidad en Crudo Pesado. Revista Tecnológica - Espol, 32(1). Recuperado a partir de http://www.rte.espol.edu.ec/index.php/tecnologica/article/view/739 


\section{INTRODUCCIÓN}

Debido a la disminución en la producción de crudo de yacimientos convencionales y al aumento en la demanda de energía alrededor del mundo a partir del petróleo, se han enfocado los esfuerzos en la producción de crudo pesado y extrapesado. Estos recursos existen en abundancia y la tecnología actual permite su explotación de una manera rentable.

El crudo pesado se caracteriza porque su grado API es menor de $22,3^{\circ}$, el extrapesado tiene API menor a $10^{\circ}$ y el crudo convencional tiene un grado API mayor a $25^{\circ}$. Del total de reservas de crudo en el mundo, el $30 \%$ corresponde a crudo convencional, $15 \%$ a crudo pesado, $25 \%$ a crudo extrapesado y $30 \%$ a arenas petrolíferas y bitumen. La viscosidad de los petróleos varía de la siguiente manera: en convencionales entre 1 y $10 \mathrm{cP}$, en pesados y extrapesados entre menos de $20 \mathrm{cP}$ y más de 1 $000000 \mathrm{cP}$; debido a la alta viscosidad que presentan estos últimos, se dificulta la producción, el transporte y la refinación [1].

La fracción pesada del crudo es la más difícil de caracterizar debido a la variedad de compuestos orgánicos de gran tamaño, cuyas estructuras químicas no se pueden identificar con facilidad. Generalmente, la caracterización de un crudo pesado se realiza a través del análisis SARA, que clasifica las fracciones del crudo en: saturados, aromáticos, resinas y asfaltenos. Los asfaltenos son la fracción más pesada y polar del petróleo e influyen de forma directa en la viscosidad del crudo, debido a sus estructuras complejas con núcleos aromáticos fusionados y nafténicos rodeados de cadenas alifáticas [2]. Se han llevado a cabo muchos estudios sobre los asfaltenos diluidos en solventes o en su medio natural y se observó que la viscosidad de las soluciones incrementa linealmente respecto a la fracción volumétrica de asfaltenos [3].

En las condiciones originales del reservorio, los compuestos ligeros y pesados del crudo son solubles y forman una sola fase líquida. Variaciones de la temperatura, presión, composición, factores mecánicos y eléctricos pueden alterar este balance en algunas moléculas y conducir a la separación en dos fases: líquida y sólida [2]. La fase sólida en el crudo está compuesta principalmente por asfaltenos precipitados, y esto tiene un impacto negativo en las operaciones habituales de la industria petrolera, en la producción dificulta la fluidez de la fase de interés y ocasiona taponamientos en el pozo, en los equipos y accesorios de superficie. En el transporte usualmente bloquea las líneas, en la refinación puede taponar las columnas de separación y desactivar los catalizadores lo que disminuye los rendimientos de refinación [4].

Yen [3] describió hace más de 50 años el fenómeno de precipitación de alfaltenos a través de la formación de agregados. La molécula elemental de asfalteno mide aproximadamente un nanómetro y puede asociarse con otras moléculas hasta formar agregados que miden hasta un micrón, es decir, 1000 veces más grandes que una sola molécula de asfalteno. Dichas asociaciones tienen lugar por formación de puentes de hidrógeno y las interacciones de los grupos polares, cuando hay suficiente concentración de partículas [5].
Investigaciones recientes describen la aglomeración de los asfaltenos en solventes o en su petróleo crudo nativo. Las moléculas de asfaltenos en concentraciones menores de $100 \mathrm{mg} / \mathrm{L}$ de tolueno se encuentran dispersas en una solución verdadera. En concentraciones mayores de 100 $\mathrm{mg} / \mathrm{L}$ y hasta $5000 \mathrm{mg} / \mathrm{L}$, los asfaltenos se aglutinan para formar nanoagregados que se dispersan en el fluido a manera de nanocoloides y se suspenden en forma estable en una fase líquida. Cuando la concentración llega 10000 $\mathrm{mg} / \mathrm{L}$, los agregados forman grupos más grandes que floculan y precipitan. Estos estudios son más difíciles de interpretar en el petróleo crudo debido a la presencia de una gran variedad de otros compuestos [6].

En los últimos años se han desarrollado tecnologías con el fin de evitar la formación de agregados y mantener a los asfaltenos suspendidos en el crudo. La inyección de aditivos químicos cumple con estos propósitos y además aporta con la reducción de viscosidad e impide la deposición de sólidos.

La dosis de los aditivos es proporcional a la tasa de reducción de viscosidad y, generalmente, va desde 200 ppm a $5000 \mathrm{ppm}$. Estos productos son solubles en petróleo, lo que permite que no se pierdan con el agua de formación y favorecen la ruptura de las emulsiones, optimizando el consumo de demulsificante. Estas múltiples acciones permiten disminuir las presiones de operación y favorecen los proceso de producción, deshidratación y transporte [7].

En el mercado se oferta una variedad de aditivos para la reducción de viscosidad, pero su aplicación se ve limitada por la selectividad de estos aditivos en función de las propiedades del crudo. Para la adecuada elección del aditivo se deben realizar pruebas de laboratorio y de campo, además de evaluar los efectos del producto [8].

El uso más frecuente de los reductores de viscosidad es para facilitar el transporte y bombeo del crudo. La inyección del químico en líneas de transporte de crudo requiere de una infraestructura diseñada a la medida, lo que puede representar una problemática por los costos de la inversión. A ello debe sumarse la adquisición del aditivo, contratación de operadores, entre otros. [9]. Esta investigación tiene como objetivo estudiar el efecto de cuatro productos comerciales en la reducción de viscosidad mediante pruebas de laboratorio para seleccionar el aditivo y la dosis con los mejores resultados. Además, la posterior aplicación del químico elegido en un pozo en la Provincia de Orellana con producción de 12000 BFPD que se transporta en una línea de $15 \mathrm{~km}$ de longitud.

\section{METODOLOGÍA}

Para el desarrollo de esta investigación se seleccionó un pozo de crudo pesado con alta viscosidad, donde la capacidad de bombeo instalada para el transporte del fluido se supera frecuentemente.

Se tomaron cinco galones de crudo por día durante tres días, y se muestreó con base a lo establecido en la norma INEN 930 Petróleo crudo y sus derivados. Muestreo. Con el fin de reducir la viscosidad del crudo, se probaron cuatro aditivos, que fueron denominados como VRX-01, VRX02, VRX-03, VRX-04.

La experimentación se desarrolló en dos fases. La primera fase consistió en la realización de análisis de 
laboratorio para la selección del reductor de viscosidad y la dosificación con los mejores resultados; el crudo empleado para estos ensayos fue previamente sometido a un proceso de decantación térmica para su deshidratación. La segunda fase correspondió al desarrollo de pruebas de campo, donde se registraron los principales parámetros de operación en la línea de transferencia de petróleo, antes y después de la aplicación del aditivo seleccionado.

\section{A. Caracterización y selección del mejor reductor de viscosidad con pruebas de laboratorio}

Por medio de ensayos de laboratorio se determinaron las principales características físicoquímicas del crudo y de los reductores de viscosidad; $y$, se evaluó la eficiencia de los aditivos al aplicarse en el crudo.

1) Caracterización del crudo: $\mathrm{Se}$ realizaron los siguientes análisis:

- Gravedad API, ASTM D1298 Standard Test Method for Density, Relative Density, or API Gravity of Crude Petroleum and Liquid Petroleum Products by Hydrometer Method (2017)

- Contenido de agua y sedimentos (BSW), ASTM D4007 Standard Test Method for Water and Sediment in Crude Oil by the Centrifuge Method (Laboratory Procedure) (2016)

- Determinación de metales, ASTM D5863 Standard Test Methods for Determination of Nickel, Vanadium, Iron, and Sodium in Crude Oils and Residual Fuels by Flame Atomic Absorption Spectrometry (2016)

- Viscosidad a $40{ }^{\circ} \mathrm{C}$, ASTM D2196 Standard Test Methods for Rheological Properties of NonNewtonian Materials by Rotational Viscometer (2018)

2) Caracterización de los reductores de viscosidad: $\mathrm{Se}$ ejecutaron los siguientes ensayos:

- Gravedad API, ASTM D1298 Standard Test Method for Density, Relative Density, or API Gravity of Crude Petroleum and Liquid Petroleum Products by Hydrometer Method (2017)

- $\quad$ pH, ASTM E70 Standard Test Method for $p H$ of Aqueous Solutions with the Glass Electrode (2015)

- El estado físico, color y olor se evaluaron como propiedades sensoriales

3) Evaluación de la eficiencia de los reductores de viscosidad: Se efectuaron pruebas con el crudo sin la adición del químico y con el uso de este; se realizó una línea base con crudo y $3 \%$ de nafta. Cada reductor de viscosidad se empleó en el crudo con concentraciones de 400, 550, 700 y 1000 ppm, y en todas las muestras también se añadió 3\% de nafta para facilitar la mezcla de los químicos con la matriz. Con las muestras preparadas se realizaron los siguientes análisis:

- Viscosidad, ASTM D2196 Standard Test Methods for Rheological Properties of NonNewtonian Materials by Rotational Viscometer (2018). Esta propiedad fue evaluada en un perfil de temperaturas de calentamiento: 40, 50, 60, 70 y $80^{\circ} \mathrm{C}$; y en un perfil de temperaturas de enfriamiento: $70,60,50$ y $40^{\circ} \mathrm{C}$. La medición de viscosidad se la realizó después de la agitación y tiempo necesario hasta que la muestra alcanzó el equilibrio térmico.

Con el reductor de viscosidad que presentó los mejores resultados en el ensayo anterior, se realizó:

- Dispersión de asfaltenos, ASTM D3279 Standard Test Method for n-Heptane Insolubles (2012). Las concentraciones para esta prueba fueron 400, 550, 700 y 1000 ppm del producto en crudo.

- Prueba de Oliensis, ASTM D1370 Standard Test Method for Contact Compatibility Between Asphaltic Materials (Oliensis Test) (2016)

\section{B. Validación de la eficiencia de los reductores de} viscosidad con pruebas de campo

Se evaluó la eficiencia del mejor reductor de viscosidad encontrado en el laboratorio, en la línea de transferencia del crudo, desde el pozo hasta una estación de procesamiento. La operadora de este bloque proporcionó las facilidades para que se realice el proceso como una alternativa de solución para problemas de transporte de crudo pesado. El proceso de validación incluyó:

1) Diseño de los sistemas de inyección de reductores de viscosidad: Con base en la dosis del reductor de viscosidad con mayor eficiencia y el volumen de crudo a tratarse, se dimensionó un tanque de inyección, capilares, una bomba de inyección, un mezclador de flujo y accesorios como válvulas, medidores de flujo y manómetros. Con el sistema de bombeo existente dentro de las instalaciones, se propuso un diagrama de tuberías e instrumentación (P\&ID).

2) Evaluación de los reductores de viscosidad en campo: Consistió en el monitoreo de las variables y condiciones operacionales en la línea de flujo antes y después de la adición del reductor de viscosidad. Las variables analizadas para la evaluación fueron:

- Temperaturas de succión y descarga en las bombas Booster

- Presión en la succión y descarga de las bombas Booster

- Viscosidad en el arribo de la línea de transferencia

- Contenido de agua y sedimentos, BS\&W, en el arribo de la línea de transferencia

- Porcentaje de emulsión del crudo en arribo de la línea de transferencia

La prueba de campo se realizó en 11 días, distribuidos como se muestra en la Fig. 1. El reductor se inyectó en dos puntos:

- Entrada de las bombas Booster (días 4 y 5)

- Manifold de entrada a la estación (días 6 y 7)

Las concentraciones del químico se establecieron en 750 y 1000 ppm por recomendación del fabricante de producto y se eligió el punto óptimo de inyección de acuerdo con los días donde se observaron mejores resultados. En los días 8 y 9, se inyectó en el punto óptimo el aditivo en la dosis teórica determinada en la fase de laboratorio. 
Durante la prueba se conservó la inyección de otros productos químicos como demulsificante, antiparafínico y anticorrosivo.

\begin{tabular}{|c|c|c|c|c|c|c|c|c|c|c|c|}
\hline & \multicolumn{3}{|c|}{ Línea Base } & \multicolumn{6}{|c|}{$\begin{array}{l}\text { Inyección del reductor de } \\
\text { viscosidad }\end{array}$} & \multicolumn{2}{|c|}{$\begin{array}{l}\text { Línea } \\
\text { Residual }\end{array}$} \\
\hline \multirow[b]{2}{*}{ 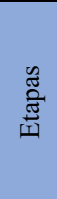 } & & & & \multicolumn{2}{|c|}{1} & \multicolumn{2}{|c|}{2} & \multicolumn{2}{|c|}{3} & & \\
\hline & & & & \multicolumn{2}{|c|}{$\begin{array}{l}\text { Inyección } \\
\text { en } \\
\text { Bombas } \\
\text { Booster }\end{array}$} & \multicolumn{2}{|c|}{$\begin{array}{c}\text { Inyección } \\
\text { en } \\
\text { Manifold }\end{array}$} & \multicolumn{2}{|c|}{$\begin{array}{l}\text { Optimiza } \\
\text { ción del } \\
\text { punto de } \\
\text { inyección } \\
\text { y la dosis }\end{array}$} & & \\
\hline Día & 1 & 2 & 3 & 4 & 5 & 6 & 7 & 8 & 9 & 10 & 11 \\
\hline$\frac{n}{0}$ & 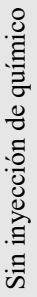 & 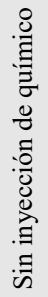 & 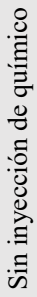 & $\begin{array}{l}\tilde{\Xi} \\
\text { : } \\
8 \\
8\end{array}$ & 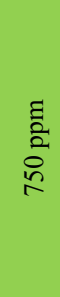 & 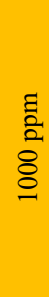 & $\begin{array}{l}\text { है } \\
\text { : } \\
\stackrel{2}{n}\end{array}$ & 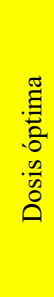 & 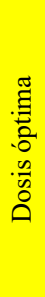 & 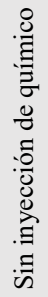 & 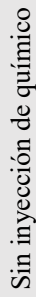 \\
\hline
\end{tabular}

Fig. 1 Cronograma de ensayo de prueba de campo

3) Evaluación de la factibilidad económica de la inyección del reductor de viscosidad: Para la evaluación económica del proyecto se desarrolló el flujo de fondos a cinco años. Se calcularon los indicadores financieros: valor actual neto (VAN), tasa interna de retorno (TIR), tiempo de retorno de la inversión, punto de equilibrio y relación Beneficio/Costo $(\mathrm{B} / \mathrm{C})$. Finalmente se elaboró un análisis de sensibilidad para mostrar la variación de los indicadores financieros respecto al precio del petróleo.

\section{RESULTADOS Y DISCUSIÓN}

A continuación, se presentan los resultados y discusión desarrollados para la prueba de productos reductores de viscosidad en el laboratorio y posterior prueba en campo.

\section{A. Caracterización y selección del mejor reductor de viscosidad con pruebas de laboratorio}

1) Caracterización del crudo: El crudo presentó un grado API de 19,5, concentración de vanadio menor a 10 ppm, concentración de níquel de 30 ppm y viscosidad de $819 \mathrm{cP}$ a $40^{\circ} \mathrm{C}$.

El contenido de agua y sedimentos en la prueba de laboratorio fue de $0,5 \%$, sin embargo, esta muestra correspondió al crudo luego del proceso de deshidratación. El BSW del fluido en la línea de producción es del 38,0 \%, por lo que se puede considerar al crudo como pesado y con una alta concentración de agua.

2) Caracterización de los reductores de viscosidad: A continuación, se describe brevemente a los reductores de viscosidad utilizados en este estudio. Por razones de confidencialidad, las identidades reales de los productos comerciales mencionados no se dan a conocer en este trabajo.

- VRX-01, promotor de flujo, contiene alquilfenol resina oxialquilada y aromáticos

- VRX-02, rompedor de emulsión e inhibidor de asfaltenos, contiene aminas etoxiladas y aromáticos
- VRX-03, inhibidor de compuestos orgánicos pesados, contienen sal de ácido orgánico

- VRX-04, rompedor de emulsión, contiene una fracción nafta disolvente, sales de potasio y ácido bencenosulfónico.

Las propiedades de los químicos reductores de viscosidad se presentan en la Tabla I. Los productos más livianos fueron VRX-01 y VRX-02. Los productos presentaron carácter ácido y se neutralizaron con amoniaco para evitar la corrosión en los sistemas de inyección de químico.

TABLA I

PROPIEDADES DE LOS REDUCTORES DE VISCOSIDAD

\begin{tabular}{|c|c|c|c|c|}
\hline Propiedad & VRX-01 & VRX-02 & VRX-03 & VRX-04 \\
\hline $\begin{array}{c}\text { Densidad a } \\
\mathbf{2 0} \\
\mathbf{}^{\circ} \mathbf{C} \\
\left(\mathbf{k g} / \mathbf{m}^{3}\right)\end{array}$ & 876,32 & 831,84 & 959,96 & 987,89 \\
\hline $\mathbf{p H}$ & 4,2 & 4,7 & 5,0 & 3,8 \\
\hline $\begin{array}{c}\text { Estado } \\
\text { físico }\end{array}$ & Líquido & Líquido & Líquido & Líquido \\
\hline Color & Claro ámbar & Ámbar & Ámbar & Ámbar \\
\hline Olor & Aromático & Aromático & Amina & Hidrocarburo \\
\hline
\end{tabular}

3) Evaluación de la eficiencia de los reductores de viscosidad: En la Tabla II se presenta datos experimentales de las pruebas de viscosidad sobre el crudo (blanco), crudo con nafta, y crudo con nafta y los diferentes aditivos en las dosis establecidas.

TABLA II

VISCOSIDAD DE LAS MUESTRAS DE CRUDO A $40^{\circ} \mathrm{C}$ CON LOS PRODUCTOS REDUCTORES DE VISCOSIDAD

\begin{tabular}{|c|c|c|c|c|c|c|}
\hline \multirow{3}{*}{$\begin{array}{c}\text { Dosis } \\
(\mathrm{ppm})\end{array}$} & \multicolumn{6}{|c|}{ Viscosidad Crudo (cP) } \\
\hline & \multirow{2}{*}{ Blanco } & \multirow{2}{*}{$\begin{array}{c}\text { Crudo + } \\
\text { Nafta 3\% }\end{array}$} & \multicolumn{4}{|c|}{ Reductor de viscosidad } \\
\hline & & & VRX-01 & VRX-02 & VRX-03 & VRX-04 \\
\hline 400 & \multirow{4}{*}{819} & \multirow{4}{*}{489} & 445 & 465 & 462 & 451 \\
\hline 5550 & & & 445 & 427 & 448 & 440 \\
\hline 700 & & & 437 & 413 & 428 & 439 \\
\hline 1000 & & & 397 & 318 & 285 & 401 \\
\hline
\end{tabular}

En la Fig. 2 y en la Fig. 3 se muestran las curvas de viscosidad a las temperaturas de calentamiento y enfriamiento, para las concentraciones de 400 ppm y 1000 ppm de aditivo en crudo, respectivamente.

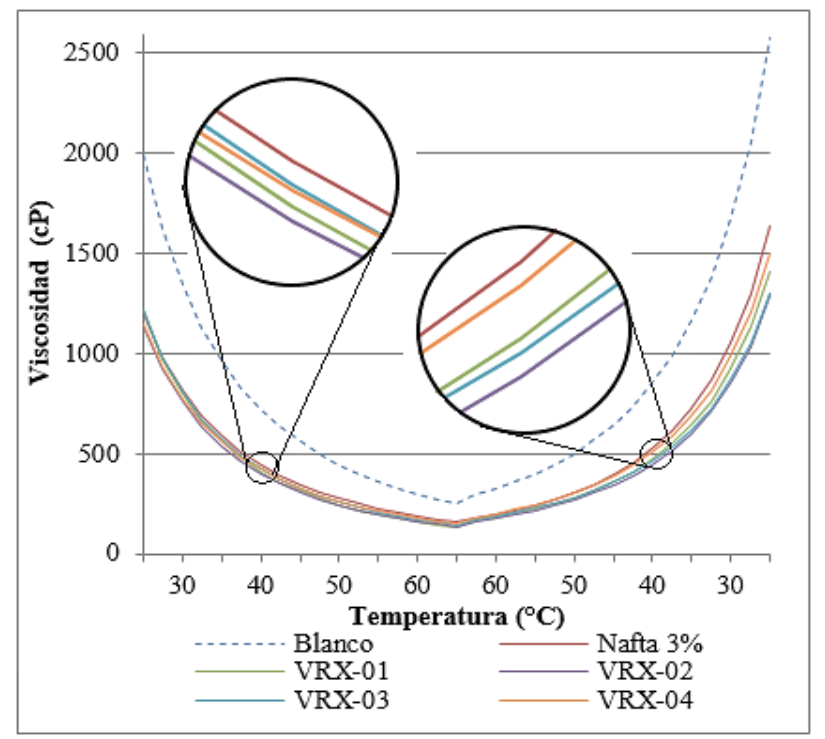

Fig. 2 Curvas de viscosidad con dosificación de 400 ppm 


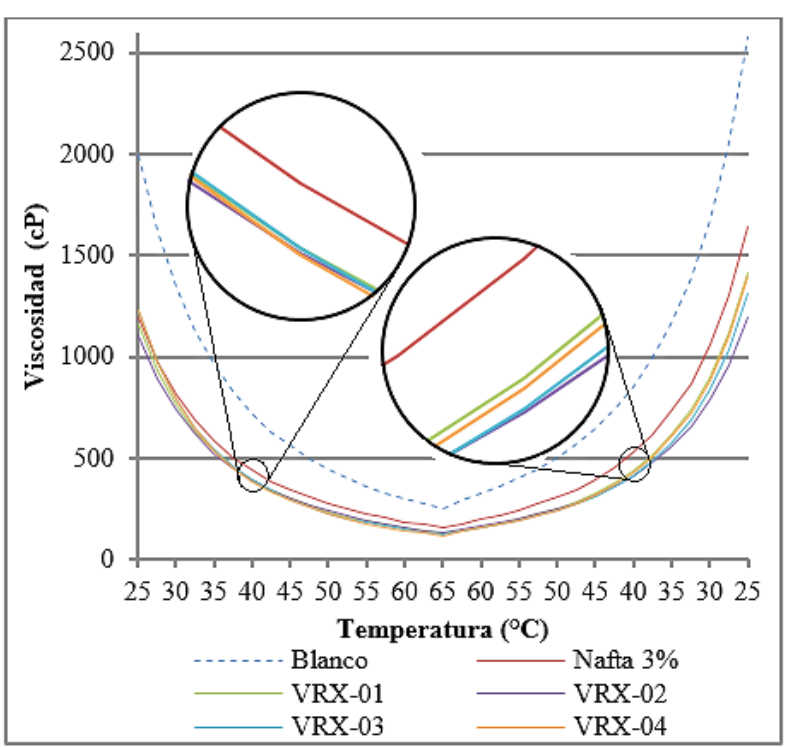

Fig. 3 Curvas de viscosidad con dosificación de 700 ppm

Entre las concentraciones de aditivo de 400 y 700 ppm, la segunda mostró mejores resultados. El químico VRX02, en una dosis de 700 ppm, logró la menor viscosidad en el crudo (413 cP a $40{ }^{\circ} \mathrm{C}$ ), y fue seleccionado para su aplicación en campo.

Para corroborar la elección de la dosis de aditivo, se evaluó la dispersión de los asfaltenos en n-heptano en las muestras de crudo sin aditivo y de crudo con reductor de viscosidad VRX-02 a las dosis antes indicadas. En la Fig. 4 se muestran los resultados de este ensayo.

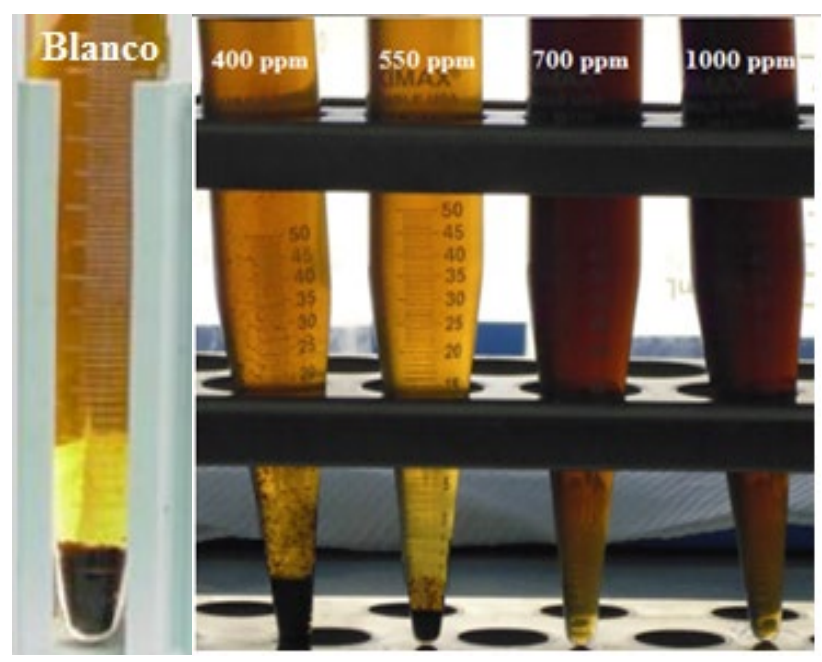

Fig. 4 Resultados del ensayo de dispersión de asfaltenos

Al colocar n-heptano en el crudo, los asfaltenos precipitan. Se observa que la fase asfalténica precipitada en el blanco y con el uso de 400 ppm del químico es similar, mientras que, con una concentración de 550 ppm, la cantidad de asfaltenos precipitados es menor. Para 700 ppm y 1000 ppm los asfaltenos se muestran dispersos en el crudo. La dispersión y la suspensión de los asfaltenos es un indicativo de la acción del químico para evitar la cercanía de las partículas, lo que previene la aglomeración y posterior precipitación de los asfaltenos [4].

Se considera que la mejor dosis del reductor de viscosidad en el crudo es de 700 ppm ya que muestra efectos similares a 1000 ppm y la cantidad de producto aplicado es menor, lo que representa menores costos.

Para estudiar la eficiencia del reductor de viscosidad VRX-02 a 700 ppm, se realizó la prueba de Oliensis o de la mancha en el crudo sin aditivos o blanco y en el crudo con el químico. Este análisis consistió en colocar gotas de mezclas de crudo, xileno y $\mathrm{n}$-heptano en diferentes proporciones sobre papel filtrante; a cada mezcla se le asigna un valor en una escala del 0 al 9. Los resultados se muestran en la Fig. 5.

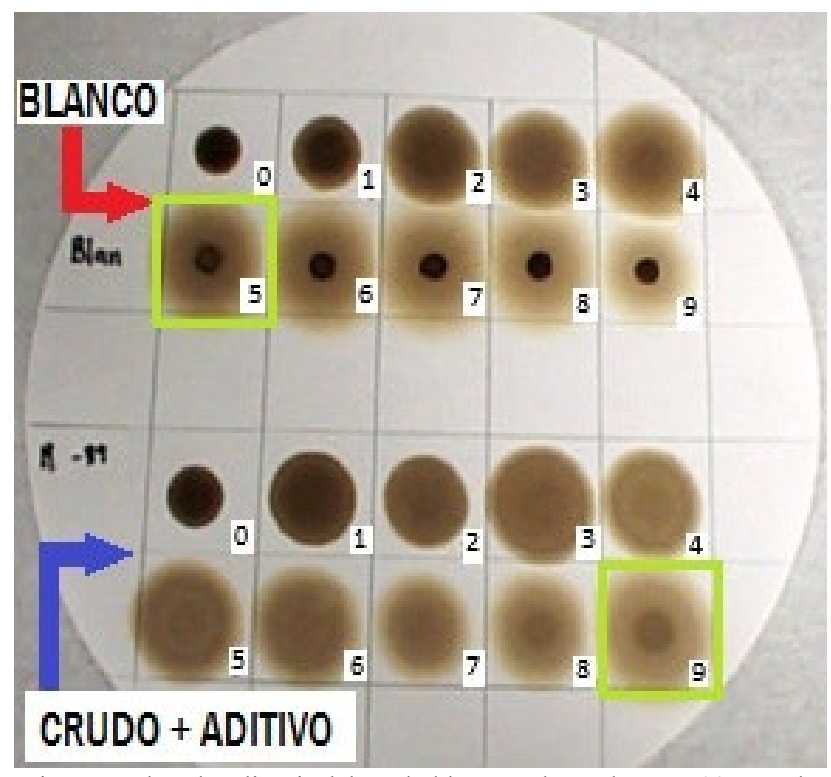

Fig. 5 Pruebas de Oliensis del crudo blanco y de crudo con 700 ppm de VRX-02

En la Fig. 5, se identificaron las manchas con presencia de una aureola o anillo. La posición de dicha mancha aporta información acerca de la estabilidad de los asfaltenos, donde siete es un valor de umbral; ubicaciones por debajo del umbral representan inestabilidad y los valores sobre este denotan asfaltenos estables.

Un comportamiento estable de los asfaltenos implica que estas partículas se mantendrán suspendidas y la probabilidad de que precipiten es reducida [11]. En la muestra del blanco, la aureola apareció en la mancha de la posición cinco, esto indica inestabilidad en los asfaltenos y su tendencia a depositarse. Después del tratamiento químico del crudo, la mancha con aureola se encontró en la casilla nueve, por lo que se determinó que la fase asfalténica logra la estabilidad, además de reducirse la viscosidad del crudo.

\section{B. Validación de la eficiencia de los reductores de viscosidad con pruebas de campo}

1) Diseño de los sistemas de inyección de reductores de viscosidad: El sistema fue diseñado con capacidad de almacenar el químico VRX-02 durante 21 días para su inyección en crudo a 700 ppm, como se definió en las pruebas de laboratorio. A continuación, se indican los parámetros más importantes del diseño:

- Tanque de almacenamiento del reductor de viscosidad (TK-01), de 5000 galones de capacidad, con las siguientes dimensiones: diámetro: 2,5 m; altura: 5,0 m; espesor: 5,0 mm 
- Bomba de inyección (P-01), con potencia 0,14 HP

- Un mezclador estático colocado en el interior de la línea de transferencia de crudo para facilitar la homogeneización con el químico

En la Fig. 6, se muestra el diagrama P\&ID del sistema de inyección.

2) Evaluación de los reductores de viscosidad en campo: La evaluación del reductor de viscosidad en campo se realizó como se indicó en la Fig. 1. Los parámetros relacionados con la operación de la bomba Booster, para la impulsión del crudo, son los más importantes a ser monitoreados. En la Tabla III se muestran los valores de las temperaturas del sistema, medidas en la succión y descarga de la bomba y al final de la línea de transferencia.

El fluido, al pasar a través de las bombas Booster, recibe energía térmica como producto del trabajo del equipo, por lo tanto, la temperatura en la descarga es mayor que en el ingreso. La temperatura de salida del fluido de la línea de transferencia es menor que en la descarga de la bomba, puesto que en el recorrido de aproximadamente $15 \mathrm{~km}$ se producen pérdidas por transferencia de calor hacia el ambiente.

TABLA III

REGISTRO DE TEMPERATURAS DEL SISTEMA

\begin{tabular}{|c|c|c|c|c|}
\hline Día & Clima & $\begin{array}{c}\text { Succión. } \\
\text { Booster } \\
\left({ }^{\circ} \mathbf{C}\right)\end{array}$ & $\begin{array}{c}\text { Descarga } \\
\text { Booster }\left({ }^{\circ} \mathbf{C}\right)\end{array}$ & $\begin{array}{c}\text { Arribo de línea } \\
\text { de transferencia } \\
\left({ }^{\circ} \mathbf{C}\right)\end{array}$ \\
\hline 1 & Lluvia & 30 & 32 & 24 \\
\hline 2 & Soleado & 35 & 37 & 32 \\
\hline 3 & Nublado & 33 & 34 & 31 \\
\hline 4 & Lluvia & 31 & 32 & 27 \\
\hline 5 & Soleado & 35 & 37 & 32 \\
\hline 6 & Soleado & 36 & 37 & 31 \\
\hline 7 & Soleado & 33 & 35 & 31 \\
\hline 8 & Lluvia & 32 & 33 & 25 \\
\hline 9 & Lluvia & 31 & 32 & 26 \\
\hline 10 & Soleado & 35 & 36 & 32 \\
\hline 11 & Lluvia & 31 & 33 & 28 \\
\hline
\end{tabular}

En cuanto a la presión, al ser monitoreada en la succión de la bomba, se obtuvo un valor de 45 psig, sin encontrarse mayores variaciones durante los días de prueba. Los resultados de la presión en la descarga de la bomba Booster se muestran en la Fig. 7.

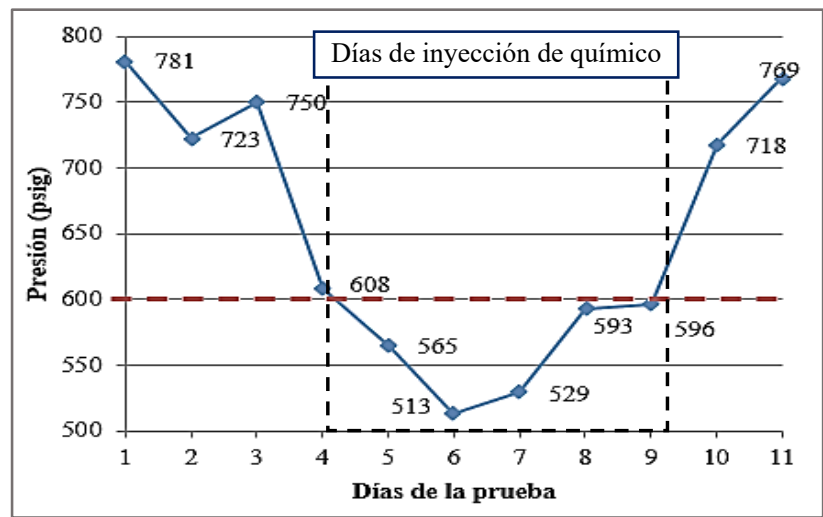

Fig. 7 Perfil de presión en la descarga de la bomba Booster

La presión máxima en la descarga de la bomba Booster es de 600 psig; sin embargo, esta presión es superada en la operación normal por lo que se rentan bombas de otras empresas de servicios para conseguir una presión adecuada para el transporte del crudo. Como se mostró en la Fig. 7, con la aplicación del producto VRX-02 los valores de presión se redujeron por debajo de 600 psig, por lo que se podrían dejar de rentar servicios externos y utilizar únicamente las bombas de propiedad de la empresa operadora.

La reducción en el trabajo que la bomba debe aportar para movilizar el fluido es resultado de los cambios en la viscosidad del crudo. En la Fig. 8 se muestran los valores de viscosidad del crudo en el arribo de la línea de transporte.

Se observó que la viscosidad varió en función de la dosis del aditivo empleada. La mayor eficiencia en la reducción

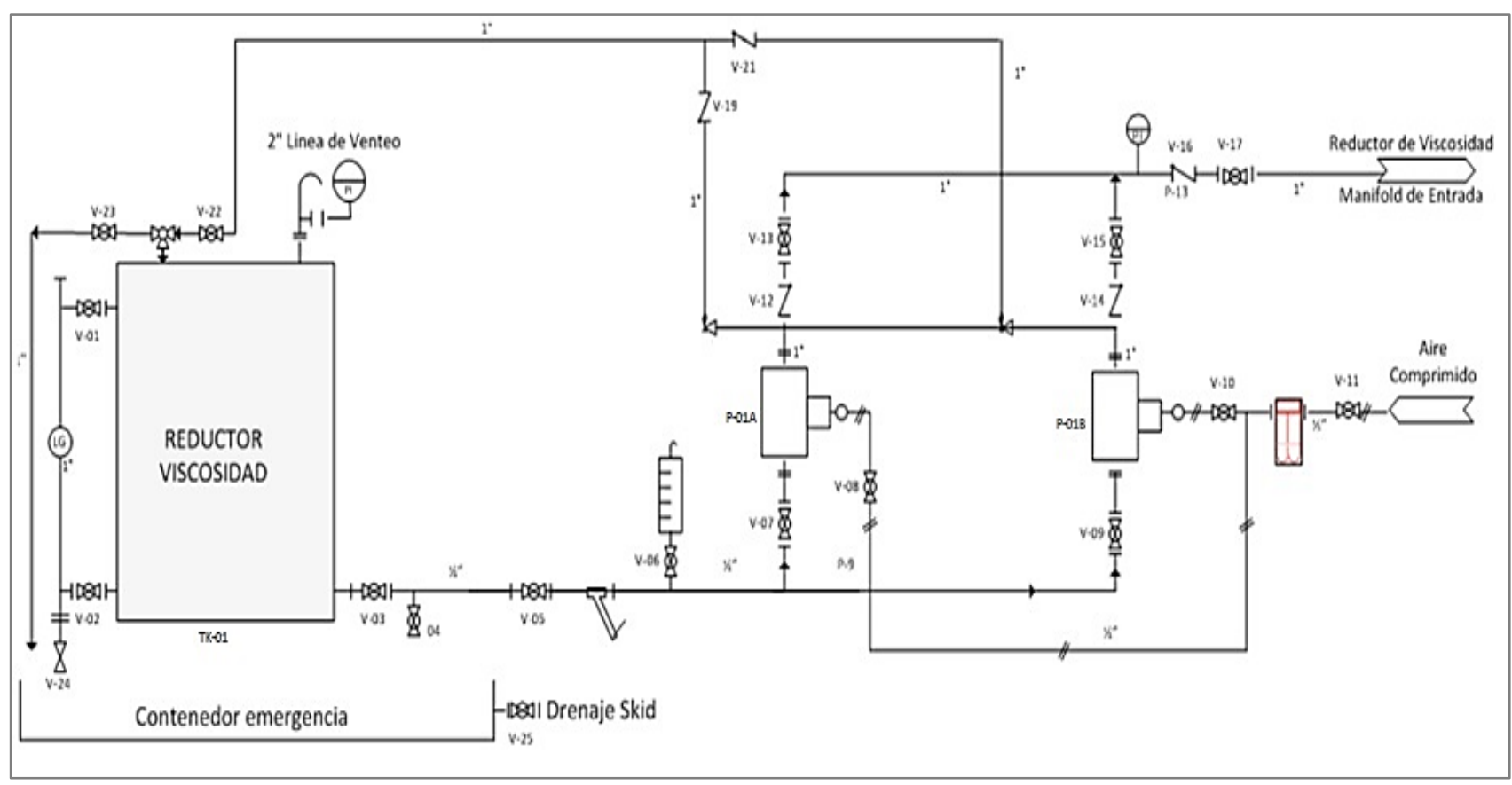

Fig. 6 Diagrama P\&ID del sistema de inyección del reductor de viscosidad VRX-02 
de viscosidad por aplicación del químico se obtuvo con la dosificación de 1000 ppm en los días 4 y 6, los valores fueron 486 y $443 \mathrm{cP}$, respectivamente.

Se esperaba que la viscosidad en los días de optimización, 8 y 9, sea semejante al día 7 (dosis de 750 ppm), sin embargo, esta propiedad incrementó su valor. Lo que pudo ser resultado de las condiciones climáticas lluviosas, pues la viscosidad depende directamente de la temperatura. Y, en estos días en el fluido, se registraron los valores más bajos: 25 y $26^{\circ} \mathrm{C}$, como se indicó en la Tabla III. A pesar de ello, se observa que la inyección del químico mantuvo viscosidades bajas del crudo respecto al fluido sin tratamiento.

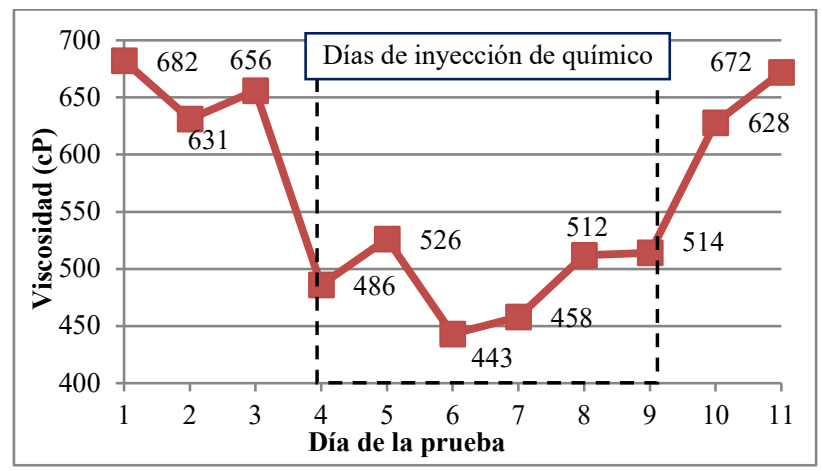

Fig. 8 Perfil de viscosidad en el arribo de la línea de transferencia de crudo

Una mayor capacidad de bombeo significa un incremento en el número de barriles de crudo transportado. Se monitoreó el volumen de fluido total transportado (crudo + agua) y el volumen de crudo sin el agua, los resultados se indican en la Fig. 9.

Con la aplicación del reductor de viscosidad, el número de barriles de fluido bombeados por día aumentó de 12000 a 12 198. En el crudo sin agua, antes del uso del aditivo, se bombearon aproximadamente 4300 BBPD, y luego de su aplicación se obtuvo 4569 BBPD. La razón de que el incremento en el número de barriles por día de crudo supere al valor de fluido total es porque el químico además actúa como rompedor de la emulsión, permitiendo que se separen con mayor eficiencia el crudo y el agua, es decir, se obtiene mayor volumen de crudo y menor volumen de agua luego de la inyección.

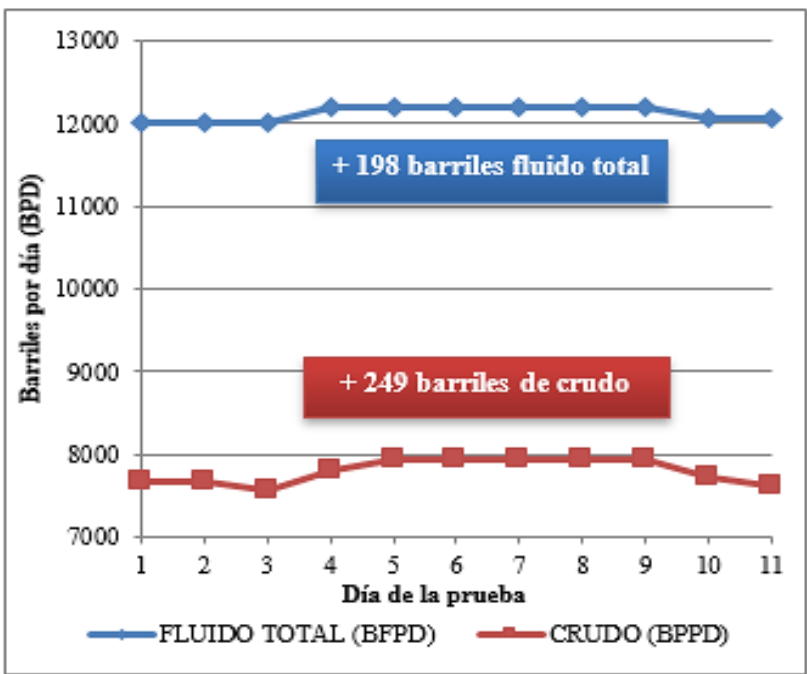

Fig. 9 Barriles de crudo y barriles de fluido total bombeado por día
Se monitorearon los valores de BSW y el porcentaje de emulsión en el crudo, los resultados se muestran en la Fig. 10. Durante la inyección se logró una reducción en el contenido de agua y sedimentos de hasta $3 \%$. El porcentaje de emulsión también disminuyó, en este caso de $8 \%$ hasta $2 \%$ al aplicarse el químico en el Manifold de entrada a estación.

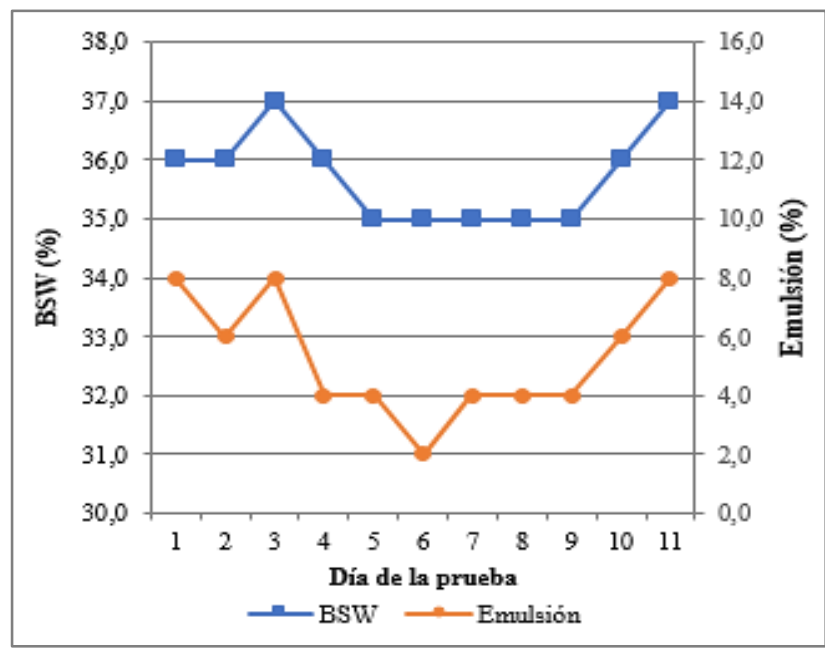

Fig. $10 \mathrm{BSW}$ y porcentaje de emulsión en el crudo en los días de prueba

La separación de crudo y del agua se relaciona con la destrucción de los agregados moleculares y la suspensión de las partículas en el fluido, ya que, por lo general en las grandes estructuras que forman los asfaltenos al unirse con otras fracciones, se encuentran atrapadas pequeñas gotas de agua [12].

En los días en los que el químico se inyectó en el Manifold de entrada a la estación se encontraron los mejores resultados, por lo que este punto fue definido como el óptimo, con la dosis de 700 ppm de aditivo que se determinó en los ensayos de laboratorio.

3) Evaluación de la factibilidad económica de la inyección del reductor de viscosidad: El análisis del flujo de fondos del proyecto, que se muestra en la Tabla IV, se realizó para un periodo de cinco años, con una tasa de crecimiento del $5 \%$ en la producción de crudo y en los costos variables. La inversión inicial del proyecto fue estimada en 133 344,76 USD para la implementación y puesta en marcha del sistema de inyección del reductor de viscosidad detallado en la Fig. 6.

Los ingresos están representados por los 298 barriles diarios de crudo que se incrementaron en la producción de la estación luego de la aplicación del aditivo; el precio del crudo en el momento de elaboración de este estudio se ubicó en 26,56 USD. En los costos fijos se incluyen los salarios del personal y alquiler de equipos, mientras que en los costos variables consta la adquisición de insumos adicionales a los empleados en la operación normal.

Los indicadores financieros del proyecto se presentan en la Tabla V. Los cálculos fueron realizados al considerar la tasa de oportunidad como la suma de una tasa pasiva referencial de 5,98\% y una tasa de riesgo del 15,0\%, en total $20,98 \%$. El VAN mostró ser mayor que 0 , el TIR mayor a la tasa de oportunidad, y la relación costo/beneficio (B/C) mayor a 1, lo que demuestra la viabilidad del proyecto en la obtención de beneficios 
económicos. El periodo de retorno de la inversión es de 0,11 años.

El punto de equilibrio, en el cual dejarían de percibirse ganancias, se encontró un precio del petróleo de 14,02 USD. El análisis de sensibilidad se realizó para estimar los valores de los indicadores financieros respecto al precio del petróleo. Los valores empleados para los cálculos fueron desde el precio del crudo en el punto de equilibrio hasta 80 USD. Los resultados se muestran en la Tabla VI.

TABLA V

INDICADORES FINANCIEROS

\begin{tabular}{|c|r|}
\hline Indicador financiero & \multicolumn{1}{|c|}{ Valor } \\
\hline Precio barril (USD) & 26,56 \\
\hline Precio barril crítico (USD) & 14,02 \\
\hline Valor Actual Neto (USD) & 3617479,20 \\
\hline Tasa Interna de Retorno (\%) & 883 \\
\hline Periodo de Retorno de la Inversión (años) & 0,11 \\
\hline Relación costo/beneficio & 1,89 \\
\hline
\end{tabular}

TABLA VI

ANÁLISIS DE SENSISBILIDAD

\begin{tabular}{|c|c|c|c|c|}
\hline $\begin{array}{c}\text { Precio del } \\
\text { crudo } \\
\text { (USD/barril) }\end{array}$ & $\begin{array}{c}\text { VAN } \\
\text { (USD) }\end{array}$ & $\begin{array}{c}\text { TIR } \\
\text { (\%) }\end{array}$ & B/C & PR \\
\hline 14,02 & 0 & 21 & 1 & 3,05 \\
\hline 15,00 & 281231,46 & 96 & 1,07 & 1,03 \\
\hline 20,00 & 1723030,79 & 438 & 1,43 & 0,23 \\
\hline 30,00 & 4610909,46 & 1117 & 2,14 & 0,09 \\
\hline 40,00 & 7498788,13 & 1798 & 2,86 & 0,06 \\
\hline 50,00 & 10386666,80 & 2480 & 3,58 & 0,04 \\
\hline 60,00 & 13274545,48 & 3162 & 4,30 & 0,03 \\
\hline 70,00 & 16162424,15 & 3846 & 5,02 & 0,03 \\
\hline 80,00 & 19050302,82 & 4530 & 5,74 & 0,02 \\
\hline
\end{tabular}

\section{CONCLUSIONES}

A través de ensayos de laboratorio se encontró que el aditivo denominado VRX.02 presentó los mejores resultados para la reducción de viscosidad del crudo en estudio, a una concentración de 700 ppm.

La dosificación del reductor de viscosidad en la línea de transferencia del crudo requirió del diseño un sistema de inyección, con un tanque de 5000 galones y una bomba de transferencia de 0,14 HP.

En la prueba de campo se determinó que el Manifold de entrada a la estación es el punto óptimo para la inyección del químico VRX.02 para la reducción de viscosidad.

La inyección del químico en la línea de transferencia logró una reducción del $28 \%$ en la viscosidad del crudo, e incrementó el volumen de fluido total bombeado en 198 BFPD y el volumen de crudo seco en 249 BOPD.

El aditivo VRX-02 a 700 ppm mostró efectos en la ruptura de la emulsión crudo-agua, por lo que se logró reducir el BSW del fluido en $3 \%$.

La evaluación económica del proyecto entregó un VAN de 3617 479,20 USD, TIR de 883\%, relación beneficiocosto de 1,89 y periodo de recuperación de la inversión de 0,11 años; lo que demuestra que la instalación del sistema de inyección es económicamente rentable.

\section{RECONOCIMIENTOS}

Este trabajo ha sido realizado con la colaboración del Laboratorio de Combustibles, Biocombustibles y Aceites Lubricantes del Departamento de Ingeniería Química de la Escuela Politécnica Nacional y la participación y soporte técnico del Ing. Orlín Montiel.

\section{REFERENCIAS}

[1] H. Alboudwarej et al., "La importancia del petróleo pesado," Oilf. Rev. Schlumberger, vol. 18, pp. 38-59, 2006.

[2] G. A. Mansoori, D. Vazquez, and M. Shariaty-Niassar, "Polydispersity of heavy organics in crude oils and their role in

TABLA IV

FLUJO DE FONDOS DEL PROYECTO

\begin{tabular}{|c|c|c|c|c|c|c|}
\hline & Año 0 & Año 1 & Año 2 & Año 3 & Año 4 & Año 5 \\
\hline $\begin{array}{c}\text { Ingresos por } \\
\text { ventas }\end{array}$ & & 2410997,28 & 2531547,14 & 2658124,50 & 2791030,73 & 2930582,26 \\
\hline Costos variables & & $-1167876,32$ & $-1226270,14$ & $-1287583,65$ & $-1351962,83$ & $-1419560,97$ \\
\hline Costos fijos & & $-67773,76$ & $-67773,76$ & $-67773,76$ & $-67773,76$ & $-67773,76$ \\
\hline \multicolumn{7}{|l|}{ Intereses } \\
\hline Utilidad bruta & & 1175347,20 & 1237503,24 & 1302767,09 & 1371294,14 & 1443247,53 \\
\hline Depreciación & & $-788,05$ & $-788,05$ & $-788,05$ & $-788,05$ & $-788,05$ \\
\hline $\begin{array}{c}\text { Utilidades antes } \\
\text { de impuestos }\end{array}$ & & 1174559,14 & 1236715,19 & 1301979,04 & 1370506,08 & 1442459,48 \\
\hline \multicolumn{7}{|l|}{ Impuestos $(0 \%)$} \\
\hline Utilidad neta & & 1174559,14 & 1236715,19 & 1301979,04 & 1370506,08 & 1442459,48 \\
\hline Depreciación & & 788,05 & 788,05 & 788,05 & 788,05 & 788,05 \\
\hline Inversiones & $-10288,23$ & & & & & \\
\hline Capital de trabajo & $-123565,01$ & & & & & \\
\hline Valor residual & & & & & & 3940,27 \\
\hline Flujo neto & $-133853,24$ & 1175347,20 & 1237503,24 & 1302767,09 & 1371294,14 & 1447187,80 \\
\hline $\begin{array}{c}\text { Flujo neto } \\
\text { actualizado }\end{array}$ & $-133853,24$ & 971521,90 & 845510,85 & 735742,83 & 640141,96 & 558414,89 \\
\hline
\end{tabular}


oil well fouling," J. Pet. Sci. Eng., vol. 58, pp. 375-390, 2007.

[3] T. F. Yen, "Asphaltenes," in Structures and Dynamics of Asphaltenes, 1st ed., O. C. Mullins and E. Y. Sheu, Eds. San Francisco: Springer Science and Business Media, 1998, pp. 120.

[4] M. Alayon, “Asfaltenos: Ocurrencia y floculación.” pp. 9-16, 2004.

[5] O. C. Mullins, E. Y. Sheu, A. Hammami, and A. G. Marshall, Asphaltenes, Heavy Oils and Petroleomics, no. 1. New York: Springer Science and Business Media, 2007.

[6] K. Akbarzadeh et al., "Los asfaltenos: Problemáticos pero ricos en potencial," Oilf. Rev. Schlumberger, pp. 24-47, 2007.

[7] A. J. Chaustre Ruiz, J. J. Ibagon Plazas, and E. A. Leon Ramirez, "New technology for flow assurance in an extra heavy oil field: Case study in the Akacias Field," in Society of Petroleum Engineers - SPE Heavy and Extra Heavy Oil Conference - Latin America, 2014, pp. 645-654.

[8] B. P. Tissot and D. H. Welte, Petroleum Formation and Occurrence, 2nd ed. Berlin: Springer-Verlag, 1978.

[9] R. Martínez-Palou et al., "Transportation of heavy and extraheavy crude oil by pipeline : A review," J. Pet. Sci. Eng., vol. 75, pp. 274-282, 2011.

[10] B. Wu and J. Gao, "A viscosity reduction study on chinese extra heavy oil by the addition of synthesized novel oil-soluble viscosity-reducing agents," Pet. Sci. Technol., vol. 28, no. 18, pp. 1919-1935, 2010.

[11] S. Ashoori, M. Sharifi, M. Masoumi, and M. Mohammad Salehi, "The relationship between SARA fractions and crude oil stability," Egypt. J. Pet., vol. 26, no. 1, pp. 209-213, 2017.

[12] I. C. V. M. Santos, P. F. Oliveira, and C. R. E. Mansur, "Factors That Affect Crude Oil Viscosity and Techniques To Reduce It: a Review," Brazilian J. Pet. Gas, vol. 11, no. 2, pp. 115-130, 2017. 